\title{
Contribuições para um Programa de Educação Continuada em Saúde Coletiva
}

\section{Reflections on a Continuing Education Program in Public Health}

\author{
Jairnilson S. Paim ${ }^{1}$ \\ Tânia Celeste M. Nunes ${ }^{2}$
}

\section{PAIM, J. S. \& NUNES, T. C. M. Reflections on a Continuing Education Program in Public Health. Cad. Salide Públ., Rio de Janeiro, 8 (3): 262-269, jul/set, 1992.}

This paper is a set of reflections for a Project on Continued Education aimed at professionals in collective health. From reflections on the subject that are being promoted by the National School of Public Health at the Oswaldo Cruz Foundation in Rio de Janeiro, the authors suggest a theoretical reference in the fields of collective health and education, pointing to the formulation of objectives based on data from the national context, socioeconomic factors, determinants of health conditions, health and labor policies, as well as structures and objectives of the home institutions of health professionals for whom the Program is designed.

This strategy points to five major aspects and recommends the creation of a data bank on graduates, articulation between universities and agencies devoted to training human resources in health, development of workshops and seminars, and analysis of local conjunctures, with the definition of likely scenarios and choices at the local level. The issue of work is the integrating thrust of the Program's actions, going beyond the academic way of teaching and learning, and from data on reality providing an educational process organized between teachers of academic institutions and agents of health practices, favouring the confrontation and solution of problems in health services as the basis for the application of knowledge that has been generated and for the reflection towards the "re-creation" of current health practices.

Keywords: Continuing Education; Human Resources; Education and Health Services

\section{INTRODUÇÃO}

Considerando-se os diferentes projetos que influenciaram doutrinariamente a formação de recursos humanos em saúde coletiva nos países desenvolvidos, há que se destacar o seu repasse para os paises dependentes que ensejaram novas questōes para a sua constituição e hegemonia (Paim, 1986).

De modo geral, tais projetos assumiram o caráter de movimentos ideológicos em que as

Departamento de Medicina Preventiva da Faculdade de Medicina da Universidade Federal da Bahia. Rua Padre Feijo, 29, $4^{\circ}$ andar, Anexo 11. Canela, Salvador, BA, 40110-170, Brasil.

2 Coordenação Nacional dos Cursos Descentralizados (Concurd). Escola Nacional de Saúde Pública. Rua Leopoido Bulhōes, 1480, 3antar, Rio de Janeiro, RJ, 21041-210, Brasil. questōes não eram colocadas no plano da ciência, através do qual fosse possível delinear um campo teórico de investigação e propiciar um aprimoramento conceitual para a apreensão das práticas de saúde. $\mathrm{Na}$ ausência deste trabatho teórico, confrontavam-se posiçôes ou visões de mundo distintas, ainda que tivessem a idéia subjacente de operar mudanças de mentalidade dos "recursos humanos" que, teleologicamente, mudariam as práticas de saúde (agentes de mudanças).

Essa crença tem-se desenvolvido intensamente entre os responsáveis pela formação desses "recursos humanos", não obstante inúmeras investigaçōes apontarem a estrutura das práticas de saúde em dada sociedade como a determinante fundamental para a definição do "perfil" desses recursos humanos. Até mesmo docentes interessados na recriação das práticas de saúde 
(em vez da sua reprodução) têm muitas vezes esquecido que a autonomia contida no aparelho escolar é relativa e que a existência de um espaço de luta político-ideológica no interior da instituição nem sempre é suficiente para produzir uma legião de criticos capaz de redefinir as práticas de saúde.

Assim sendo, a visão parcializada desses profissionais, formados com uma consciência crítica para atuarem como "agentes de mudança", deve ser substituída por uma proposta que entenda os limites dessa "mudança", se efetuada por consciências individuais. Mudanças substantivas, politicamente viáveis, tendem a se realizar pela ação conjunta de indivíduos articulados com movimentos sociais de sustentação (associativos, sindicais ou comunitários), desde quando balizadas por uma teoria que dê conta das lutas sociais pela transformação de estruturas.

A evolução e crescente complexificação das práticas que organizam o trabalho em saúde, com a incorporação de novas tecnologias em ritmo acelerado, vêm estabelecendo padrōes mutáveis do trabalho nesse campo, envelhecendo rapidamente os cenários estabelecidos para a sua realização. Convivendo com essa realidade, o mundo das comunicações também coloca à disposição das estruturas de ensino meios modernos cada vez menos escolares, facilitando a comunicação com os profissionais que compõem a força de trabalho do setor.

Essas reflexōes se tornam fundamentais para orientar a formação de especialistas em saúde coletiva. Nesse particular, Costa (1988) oferece indicaçōes relevantes para a reorientação de especialistas em saúde coletiva face à Reforma Sanitária. É o caso, por exemplo, da identificação de lacunas nos cursos quanto à qualificação para o desenvolvimento gerencial $\mathrm{e}$ em outras áreas específicas e do destaque dos elementos relevantes para a montagem de programas de educação continuada.

Nesse sentido, a concepção de que o processo educacional se dá de forma gradual e complementar, em cada uma de suas fases, é fundamental para estabelecer níveis crescentes de capacitação, que podem iniciar numa proposta mais generalista. A formação, nesse caso, se daria ao longo da vida profissional, alimentando-se das inovações tecnológicas e da recriação das práticas, cujos conteúdos próprios seriam também gerados desse processo contínuo.

A busca de complementos educacionais mais especializados seria uma definição decorrente da relação profissional/trabalho e da maturidade alcançada no desempenho dessa atividade, seja pelo movimento pessoal do trabalhador, seja pela recomposição das práticas onde ele estaria inserido.

O presente artigo representa um detalhamento de um trabalho anterior (Ministério da Saúde, 1990) que estabeleceu um ordenamento preliminar de idéias, conceitos, concepçōes, proposiçōes e estratégias passíveis de fundamentar a elaboração de um Programa de Educação Continuada em Saúde Coletiva. Resulta de uma preocupação da Escola Nacional de Saúde Pública (Ensp), através da Coordenação Nacional dos Cursos Descentralizados (Concurd), que tem promovido discussões sobre o tema no sentido de estimular a formulação de um programa dessa natureza. Esse trabalho abre a perspectiva de uma linha contínua de reflexão/ação, representada pela Ensp e pelo conjunto de universidades inseridas na pósgraduação em saúde coletiva no Brasil e também pelas expressões de realidades de saúde. seja na produção de serviços ou em outros espaços onde esse saber será objeto de interesse de profissionais e população.

Trata-se, portanto, de um texto aberto a contribuições, inspirado no produto inicial de um trabalho coletivo. Não reproduz integralmente o rico conteúdo das discussões do grupo precursor constituido pela Ensp/Fiocruz, ainda que procure reter suas idéias centrais. Traz, possivelmente, vieses de análise e de interpretação dos autores, mas que podem ser superados através de debates criticos e sugestões. Nesse sentido, o presente artigo visa discutir e sistematizar principios, objetivos e estratégias para a formulação de um Programa de Educação Continuada em Saúde Coletiva (PEC-SC) coerente com a proposta da Reforma Sanitária.

\section{PRINCÍPIOS}

Ao se considerar os momentos constitutivos da prática em saúde coletiva (objeto, meios de trabalhos e atividades), as relações técnicas e 
sociais, integtrantes desse trabalho e as organizaçōes em que se realiza, a presente proposta assenta-se nos seguintes principios:

- A educação é um processo contínuo;

- Todo grupo social é educativo;

- O esforço educativo é universal;

- A educação permanente é integral;

- A educação é um processo dinâmico;

- A educação é um processo ordenador do pensamento;

- O sistema educativo tem caráter integrador;

- A educação é um processo inovador;

- O conhecimento se origina nas necessidades ou problemas sociais dentro de um projeto histórico;

- O PEC descentralizado reconhece a autonomia, rejeita a tutela e inclui uma ou mais instituições de referência nacional, aqui representada pela Ensp/Fiocruz;

- O PEC é flexível, pois comporta retificaçōes e ajustes no processo;

- O PEC é participativo no planejamento, execução, acompanhamento e avaliação;

- O PEC toma as práticas de saúde coletiva como eixo integrador;

- O PEC assume os problemas de saúde e dos serviços enquanto geradores de áreas temáticas.

\section{OBJETIVOS}

Tendo em vista o marco de referência e os principios explicitados, um Projeto de Educação Continuada em Saúde Coletiva (PEC-SC) buscaria alcançar os seguintes objetivos:

a) Articular instituições acadêmicas e órgãos de desenvolvimento de recursos humanos com organizações responsáveis pela atenção à saúde, visando o planejamento conjunto da formação e utilização dos agentes das práticas de saúde coletiva;

b) Desenvolver experiências pedagógicas no âmbito dos serviços de saúde que facilitem a reflexão sobre as práticas de saúde empreendidas e sua reatualização, considerando as mudanças no perfil epidemiológico, na organização dos serviços de saúde e na consciência sanitária e ecológica dos cidadãos;

c) Identificar, continuamente, necessidades de saúde, problemas e opçōes para o desenvolvimento dos serviços, orientar análises de situaçōes e programar atividades de capacitação; d) Apoiar o desenvolvimento da qualificação profissional dos egressos dos cursos de pósgraduaçāo em saúde coletiva, visando a formação de quadros atualizados e solidários ao projeto da Reforma Sanitária;

e) Contribuir com a implantação das diretrizes do Sistema Único de Saúde, de acordo com a Constituição da República e as Leis 8080/90 e $8142 / 90$ (2), particularmente a descentralização, o atendimento integral e a participação da comunidade no esforço de reorganização das Secretarias Estaduais e Secretarias ou Departamentos Municipais de Saúde;

f) Difundir informações atualizadas no campo da saúde coletiva para os egressos dos respectivos cursos de pós-graduação, desenvolvendo estratégias pedagógicas que estimulem e facilitem a discussão e apropriação desses conhecimentos pelos demais agentes das práticas de saúde inseridos nas instituições públicas, especialmente no nível local;

g) Formular, de modo descentralizado e participativo, objetivos educacionais, planos de estudos e procedimentos de acompanhamento e avaliação, considerando as áreas temáticas básicas do campo da saúde coletiva (Epidemiologia, Planejamento e Administração de Saúde, Ciências Sociais em Saúde, Educação em Saúde e Medidas de Prevenção e Controle de Doenças e Agravos);

h) Viabilizar, em tomo dos temas de saúde coletiva, a participação de todos os profissionais de saúde envolvidos na prestação de serviços, em niveis compatíveis com cada atividade, respeitando a caracteristica de cada um;

i) Implantar e manter um banco de dados com informações atualizadas sobre egressos dos cursos de pós-graduação em saúde coletiva, sua trajetória institucional e profissional, bem como sobre o contex to socioeconômico e político em que estão inseridos tais trabalhadores.

\section{ESTRATÉGIAS}

A formação de um banco de dados sobre egressos constitui-se numa primeira estratégia para o desenvolvimento de um programa dessa 
natureza. Através desse banco de dados, seria possível dispor, de forma continuada, de informações para a identificação de necessidades de qualificação, acompanhamento, avaliação, além do inventário de atividades desenvolvidas no campo da saúde coletiva e os distintos "perfis profissionais" desses egressos. Essas informaçōes deverão ser complementadas com dados de contexto, face à "necessidade de interpretar corretamente os fatos e fenômenos que caracterizem o cenário em que o processo educativo está inscrito (Souza, 1981). Questōes relacionadas ao mercado de trabalho e à política de pessoal das instituições, no que se refere aos profissionais de saúde coletiva, são de grande relevãncia para balizar as táticas do projeto. Contextos em que predominam a desvalorização do serviço público, o achatamento salarial dos "sanitaristas", o desvirtuamento dos planos de carreira etc. podem redirecionar a busca de formas alternativas de remuneração ou simplesmente a migração desses profissionais para outros postos do mercado de trabalho em saúde. Quanto aos que permanecem nos serviços públicos, podem não estar motivados para a sua qualificação profissional diante de falta de perspectivas imediatas de melhoria de remuneração ou de realização de trabalho gratificante. Em contextos dessa natureza, a formulação e implementação do PEC-SC devem submeter-se a uma rigorosa análise política e estratégica. Como adverte Santana, o sujeito da educação continuada nos serviços de saúde é uma pessoa adulta, um trabalhador - servidor público com uma visão de mundo (formação ideológica) e uma inserção socioeconômica (classe social). "Conhecer a natureza de cada uma destas características é uma precondição indispensável para a formulação e execução de propostas de Educação continuada nos serviços de saúde" (Santana, 1990).

Na medida em que um PEC-SC não se limite à transmissão de informações (atualização), mas vise propiciar o desenvolvimento profissional integral dos agentes das práticas de saúde coletiva na perspectiva da democratização torna-se imprescindível uma discussão tanto mais ampla e profunda quanto possível com tais sujeitos. Como destaca Muller, a educação permanente se produz não só quando a instituição organiza atividades de capacitação, e sim quando o pessoal, convencido da vantagem de estar atualizado e motivado para lutar por sua própria imagem profissional e pessoal, assim como pela da equipe de saúde a que pertence, busca opções para alcançar a informação e a formação que necessita, seja por meio da consulta a outros colegas, da investigação bibliográfica ou da colaboração no desenvolvimento de programas educativos no nível local" (Muller, 1987). Tal comentário se apresenta bastante pertinente para a análise da situação atual dos egressos dos cursos de saúde pública inseridos nos serviços. Não cabe, portanto, a formulação de um programa em bases idealistas nem a sua implementação em moldes patemalistas. Cumpre problematizar com os egressos a sua situação real nos serviços de saúde, bem como as representações que elaboram sobre tal situação e as perspectivas. É recomendável, como segunda estratégia, a realização de seminários e oficinas de trabalho, com a participação da clientela potencial, para levantar temas de interesses através da problematização do exercicio do trabalho dos agentes das práticas de saúde, na medida em que forem sistematizados os resultados do "estudo de egressos" e se obtenham bases de apoio do marco conceitual do projeto.

A terceira estratégia diz respeito às articulaçōes entre instituições acadêmicas e de serviços de saúde, no âmbito estadual, visando a sensibilização de docentes a serem envolvidos no PEC-SC. Nessa perspectiva, o "Programa de Capacitação de Docentes dos Núcleos Descentralizados", proposto pela Concurd, poderia constituir-se num projeto dinamizador (estratégico) do PEC-SC. Tais docentes comporiam "equipes facilitadoras" ao nivel dos Estados, contribuindo com análises de situaçōes, identificação de necessidades de formação e discussão de temas e problemas em oficinas de trabalho e seminários.

A perspectiva de envolvimento de instituições acadèmicas, no âmbito dos Estados, poderá ampliar o universo de egressos, ou seja, exalunos da Ensp e de outras escolas de Saúde Pública e centros de pós-graduação em saúde coletiva vinculados às universidades. Assim, o programa poderá ganhar em abrangència, obtendo parceiros relevantes, tal como ocortera, positivamente, na experiência do PAR e no 
modelo dos cursos CAPSIS e CADRHU (Paim, 1986).

Por tratar-se de uma iniciativa pioneira numa conjuntura com limitações de recursos, o PEC$S C$ não atingiria, num primeiro momento, os serviços como um todo e dos demais profissionais de saúde. Tendo a sua clientela centrada nos egressos dos cursos de pós-graduação em saúde coletiva, deveria empregar técnicas e meios instrucionais que envolvessem trabalho de grupo, podendo, desse modo, ampliar o seu objeto de atuação. Assim, a realização de eventos intencionalmente participativos e nãoexclusivos dos profissionais de saúde coletiva seria fundamental para a extensão da cobertura do projeto.

O reconhecimento da diversidade dos "Brasis" e das especificidades regionais e locais impede uma definição prévia das áreas e conteúdos temáticos. A análise da situação de saúde e a discussão de cenários a médio prazo poderão balizar o recorte do programa quanto às ênfases nos problemas de saúde, gerenciais e políticoinstitucionais. Assim, a quarta estratégia constituir-se-ia no balanceamento dessa análise da situação com as informaçōes organizadas nas etapas anteriores e sobre o modelo de capacitação. De qualquer nodo, é possível explicitar a preferência por um recorte que privilegie problemas identificados, em vez de disciplina e/ou especialidades da saúde coletiva. Assim, ao invés dos conteúdos serem organizados por pacotes de disciplinas (Epidemiologia, Estatística, Planejamento etc.), poder-se-ia optar por uma combinação de conteúdos para o enfrentamento de problemas priorizados. A metodologia utilizada poderia partir da análise de "situaçōes-problemas" e processos gerados no àmbito dos serviços de saúde para apoiar o desempenho do profissional na realização de a tividades da sua função, segundo o nível de inserção na instituição (serviço, estabelecimento, distrito sanitário, região, nivel central etc.). A opção por um "modelo de capacitação", no entanto, dependeria da análise de situação de cada Estado, considerando a participação das instituições acadêmicas e de serviços a esse nível, sobretudo de agentes envolvidos com planejamento, normatização, supervisão e auditoria.

$\mathrm{Na}$ Figura 1, encontra-se como sugestão preliminar um conjunto de áreas temáticas consideradas relevantes para a organização do SUS, especialmente no que se refere ao apoio aos processos de municipalização e distritalização.

A ênfase no nível local não significa uma opção ideológica pelo basismo ou subordinação a particularismos. Trata-se da escolha de uma quinta estratégia no sentido de contribuir com a apropriação de poder técnico pelos agentes inseridos em instâncias fundamentais para a diretriz política da descentralização.

Em síntese, a proposta metodológica subjacente às cinco estratégias indicadas apresenta identidades com certas iniciativas promovidas pela OPS no sentido de reconceituar os processos de capacitação do pessoal empregado nos serviços de saúde. Para tanto, poder-se-ia adotar na análise as seguintes categorias: o contexto dos processos de capacitação, a identificação das necessidades de aprendizagem, as características do processo educativo e o monitoramento e seguimento do mesmo (Haddad, 1987). Através desse enfoque e utilizando-se algumas indicações contidas na Figura 1, o programa poderia ser detalhado em termos de operações e ações.

\section{PROPOSTA METODOLÓGICA}

A educação continuada, por centrar-se sobre as práticas de saúde, por enfrentar problemas não estruturados no âmbito dos serviços e nas comunidades e por privilegiar as oportunidades educativas surgidas no cotidiano dos trabalhadores de saúde, escapa da concepção habitual da planificação educacional. Daí alguns autores admitirem ações de oportunidades "não-planificáveis" nessa planificação (Haddad, 1987).

Ainda que não seja recomendável um detalhamento do caráter normativo, alguns passos poderiam ser delineados para uma melhor apreensão do caminho a seguir. "Pensar para agir e agir para pensar melhor" (Haddad) continua sendo um ensino fundamental para os planejadores e uma singela indicação da relação teoria-prática. Nesse sentido, o esquema proposto por Haddad et al. para orientar o processo de educação continuada poderia servir de referência inicial (Haddad, 1987). Assim, a partir da 


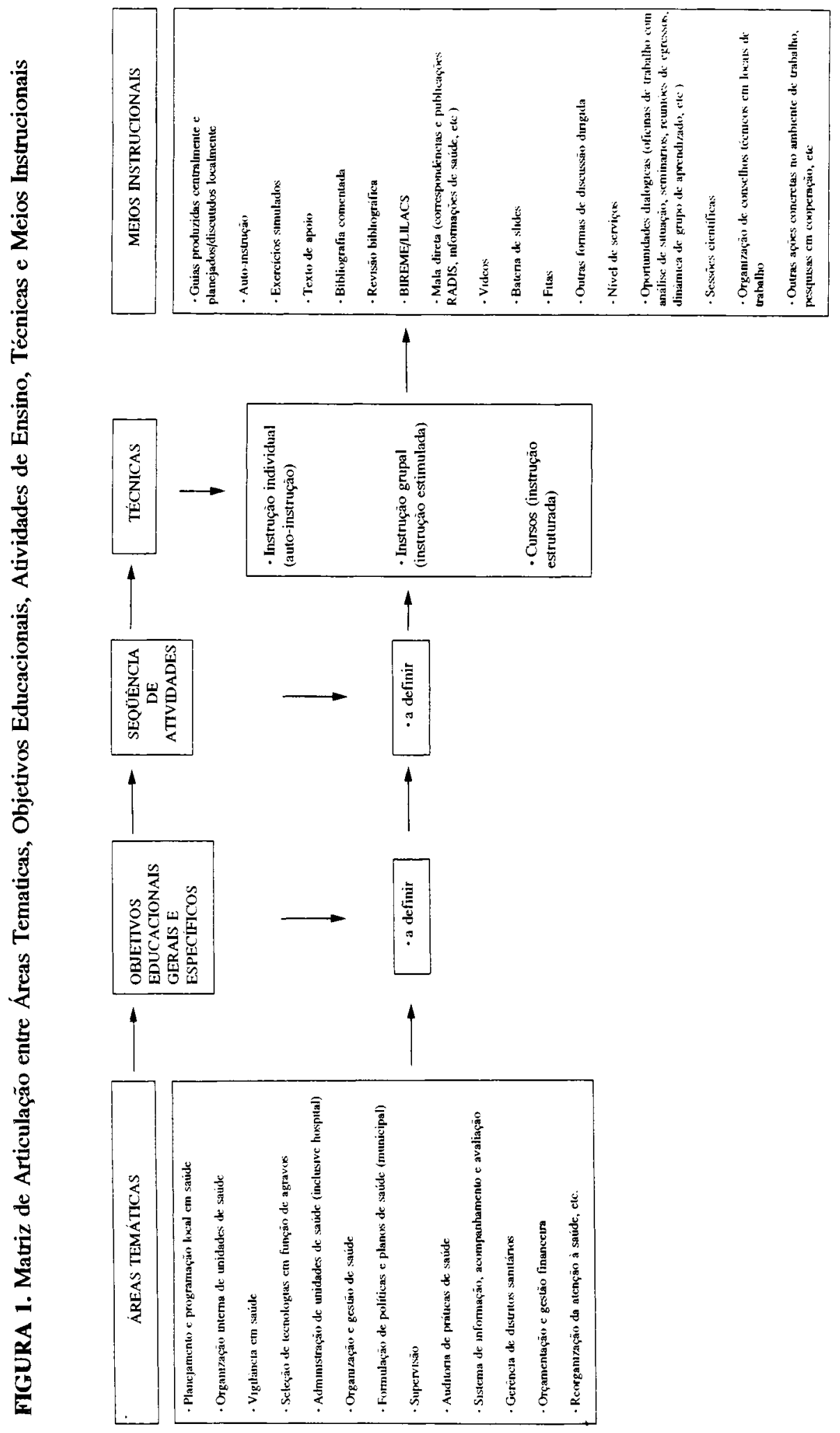


análise do contexto em que se examina a situação nacional, os fatores sociais e econômicos determinantes da situação de saúde, as políticas de saúde e trabalho, as estruturas e objetivos das instituições, seriam definidos os objetivos do programa de educação continuada. Diante de prováveis cenários e da situação-objetivo, seriam estabelecidas opções através das quais se indicariam alternativas para desenvolver o processo de capacitação.

$O$ segundo momento envolveria a identificação de necessidades de aprendizagem, que passa pelo "estudo de egressos", pela formação do banco de dados e pela realização de seminários e oficinas de trabalho com a clientela potencial. Tratam-se de necessidades sociais, epidemiológicas, políticas, administrativas, técnicas e individuais que devem ser analisadas e discutidas em cada situação concreta.

Os perfis educacionais constituem-se num terceiro momento, onde seriam relacionados conhecimentos e habilidades para enfrentar necessidades de aprendizagens, solicitações dos serviços, demandas sociais e aspirações individuiis. Ao contrário da educação tradicional, "que estabelece, a priori, momentos determinados para a aprendizagem" (Haddad, 1987), no caso da educação continuada, tais conhecimentos e habilidades "poderão adquirir-se gradual e progressivamente, segundo se apresenta um problema relacionado com o objetivo educacional" (Haddad, 1987).

Finalmente, no que se refere ao processo de capacitação (quarto momento), buscar-se-ia assumir o trabalho ou, mais especificamente, as práticas em saúde coletiva como eixo integrador do processo educativo. Reuniōes de equipe, sessōes técnico-científicas, organização de conselhos ou comissões técnicas (com representartes das diversas categorias de trabalhadores de saúde) e outras ações concretas no ambiente de trabalho (levantamento, pesquisas em cooperação etc.) podem representar iniciativas e oportunidades para instrumentalizar o trabalho como eixo educativo, privilegiando o enfrentamento $e^{f}$ a solução de problemas. Estas ações deveriam ser complementadas com outras modalidades de trabalho educativo, como a supervisão capacitante, orientação de estudos, auto-instruçōes e modelos de educação a distância etc. Diante da amplitude e complexidade de técnicas e meios instrucionais passiveis de utilização (Figura 1), caberia definir de forma mais clara a administração desse processo de capacitação, especificando as competências das instituiçōes acadêmicas consorciadas e dos serviços de saúde nos seus diversos níveis. $O$ monitoramento e o seguimento, uma vez concebidos e montados, auxiliados pela supervisão capacitante, pela análise de desempenho e por investigações educacionais operativas, possibilitariam as correções e ajustes do programa e, particularmente, do processo de capacitação.

\section{COMENTÁRIOS FINAIS}

A formulação e implementação de um Programa de Educação Continuada em Saúde Coletiva, além de uma aspiração de várias instituiçōes acadêmicas e de serviços de saúde, representam uma necessidade imperiosa diante das alterações do perfil epidemiológico e dos problemas de organização dos serviços de saúde no Brasil.

Mesmo considerando os custos de um empreendimento como esse, existem condições capazes de favorecer uma racionalização de recursos disponíveis no âmbito acadêmico e nas instituições de saúde e, conseqüentemente, possibilitar uma implantação gradual em áreas estratégicas. A existência de escolas de saúde pública, de caráter nacional e macrorregional, e de centros de pós-graduação em saúde coletiva, além de departamentos de medicina preventiva e social ou similares, proporciona a base para a construção de uma rede responsável pelo PECSC. Do mesmo modo, o apoio de outros organismos e entidades como a OPAS/OMS, Abrasco, Alaesp, Alames etc., poderia facilitar a mobilização de vontades e de recursos para a implementação de ações educativas consistentes com o projeto da Reforma Sanitária.

\section{AGRADECIMENTOS}

Os autores registram o seu agradecimento especial aos demais membros da "equipe precursora que, reunida em Salvador de 01 a 02 de outubro de 1990, discutiu o objeto, a concepção e as linhas de atuação do Projeto de Educação Continuada: Joaquim Alberto Cardoso de Melo (Ensp); Maria das Graças Soares Costa (Ensp); 
Miriam Struchiner (Ensp); Pedro Miguel Santos Neto (Nesc-PE); Rosália Maria Ferreira Moura (Nesc-RN); Maria Cristina Illanes Valenzuela (ESP-RS) e Norma Carapiá Fagundes (Sesab).

\section{RESUMO}

PAIM, J. S. \& NUNES, T. C. M. Contribuiçōes para um Programa de Educação Continuada em Saúde Coletiva.

Cad. Saúde Públ., Rio de Janeiro, 8 (3): 262 269, jul/set, 1992.

$O$ presente trabalho constitui-se em um conjunto de reflexões para um Projeto de Educação Continuada dirigido a profissionais de saúde coletiva. A partir de reflexões sobre o tema que vêm sendo estimuladas pela Escola Nacional de Saúde Pública da Fiocruz, os autores sugerem um referencial teórico nos campos da saúde coletiva e da educação, apontando para a formulação de objetivos baseados em dados do contexto nacional, em fatores sócio-económicos, nos determinantes das condições de saúde, nas políticas de saúde e trabalho e nas estruturas e objetivos das instituições onde estejam inseridas as clientelas eleitas pelo Programa.

A estratégia aponta para cinco pontos fundamentais, recomendando a organizaçāo de um banco de dados de egressos, a articulação entre universidades e órgãos formadores de recursos humanos da área de saúde, a realização de oficinas e seminários, a análise de conjunturas locais com definição de seus prováveis cenários e a opção pelo nível local. O Programa elege o trabalho como eixo integrador de suas ações, superando o modo escolar de ensinar e aprender, oferecendo um processo educativo a partir de dados da realidade, organizado entre docentes das instituições acadêmicas e agentes das práticas de saúde, privilegiando o enfrentamento e a solução dos problemas dos serviços como referência para a aplicação dos conhecimentos produzidos e base de reflexão para a recriação das práticas de saúde em vigor.

Palavras-Chave: Educação Continuada; Recursos Humanos; Educação em Serviços de Saúde

\section{REFERÊNCIAS BIBLIOGRÁFICAS}

CABRAL, A., 1969. Palavras de ordem geral. Edição da Direção Regional de S. Vicente, p. 7.

CONSELHO NACIONAL DE SECRETÁRIOS MUNICIPAIS DE SAÚDE, 1990. Sistema Único de Saúde. CONASEMS. Brasilia: Publicações Técnicas, $\mathrm{n}^{\circ} 2$, dezembro, 26p.

COSTA, M. G. S., 1988. A prática e a formaçāo do Sanitarista. O Casu de Alagoas. 1979-1986. Tese de Mestrado, Salvador: Universidade Federal da Bahia.

HADDAD, J.; MOJICA, M. J.\& CHANG, M., 1987. Organização Pan-americana da Saúde. Processo de educación permanente en Salud. Educación Médica y Salud, 21: 11-29.

MINISTÉRIO DA SAÚDE \& FIOCRUZ / ENSP / CONCURD, 1990. Projeto de Educação Continuada. Reunião com equipe precursora - 1 a 2.10.90. Súmula, 13p. (Mimeo)

MULLER, A. K., 1987. Organização Pan-americana da Saúde. La educación a distancia como opción metodológica para el desarollo de processos permanentes de educación para los recursos humanos en Salud. Educación Medica y Salud, 21: 56-59.

PAIM, J., 1986. A formação de recursos humanos em Saúde Coletiva. In: Saúde, Crise e Reformas, pp. 105-129, Salvador: Centro Editorial e Didático da Universidade Federal da Bahia.

RODAS, I;; QUINONEZ, J.; LOPEZ, M. T. \& MOLINA, S., 1989. Organização Pan-americana da Saúde. La facilitación pedagógica de los responsables de la educación permanente. Educación Médica y Salud, 23: 283-295.

SANTANA, J. P., 1990. Educação continuada nos serviços de saúde. Boletim da Associaçāo Brasileira de Educação Médica, 22: 2.

SOUZA, A. M. A.; GALVÃO, E. A. \& SANTOS, I., 1981. El Proceso Educativo. In: Educación Permanente de Personal de Salud en la Región de las Americas, Serie Desarrollo de Recursos Humanos, vol. 4, Washington, D. C.: Organização Pan-americana de Saúde.

, 1981. Introdución: revisiones previas necesarias. In: Educación Permanente de Personal de Salud en la Región de las Americas, Serie Desarrollo de Recursos Humanos, vol. 4, pp. 3 20, Washington, D. C.: Organização Pan-americana de Sauide. 